\title{
First Principles Calculation of Optical Properties of Transition Metals for Surface Plasmon Resonance Application
}

\author{
Muhammad Arifin,,${ }^{\mathrm{a}, \mathrm{b}} \uparrow{ }^{\dagger}$ Takahiro Matsumoto, ${ }^{\mathrm{a}}$ Abdul-Muizz Pradipto, ${ }^{\mathrm{c}}$ Toru Akiyama, ${ }^{\mathrm{a}}$ Tomonori Ito, ${ }^{\mathrm{a}}$ \\ Kohji Nakamura ${ }^{\text {a }}$ \\ ${ }^{a}$ Department of Physics Engineering, Mie University, 1577 Kurimamachiya-cho, Tsu city, Mie 514-8507, Japan \\ ${ }^{\mathrm{b}}$ Department of Physics, Universitas Gadjah Mada, Sekip Utara BLS 21, Yogyakarta 55281, Indonesia \\ ${ }^{c}$ Faculty of Mathematics and Natural Sciences, Institut Teknologi Bandung, Jl. Ganesha No. 10, Bandung 40132, Indonesia \\ ${ }_{\dagger}$ Corresponding author: 419db01@m.mie-u.ac.jp
}

Received: 13 December, 2019, Accepted 29 March, 2020, Published 9 April, 2020

Optical properties of several transition metals, namely $3 \mathrm{~d}$ (Fe, $\mathrm{Ni}, \mathrm{Co}, \mathrm{Cu}), 4 \mathrm{~d}(\mathrm{Ru}, \mathrm{Rh}, \mathrm{Pd}, \mathrm{Ag})$, and 5d (Os, Ir, Pt, Au) metals, for the application of surface plasmon resonance (SPR) were investigated. Using first-principles density functional theory method, we calculated the optical conductivities and the dielectric constant by including contributions from intraband and interband transitions. The calculated results for all systems reproduce the experimental trends qualitatively. The contribution of the Drude model is very dominant at low energy levels and
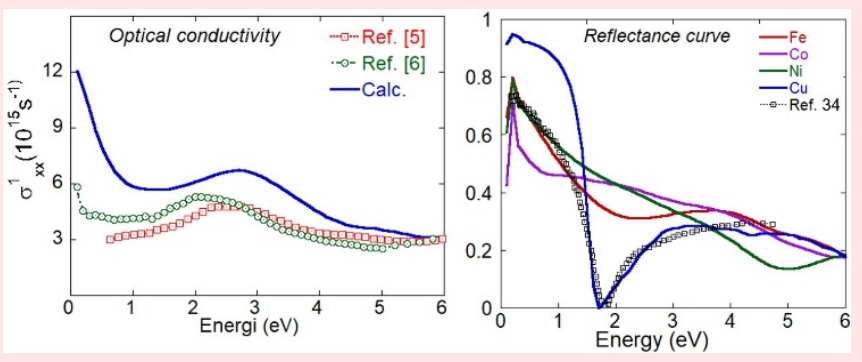
gives a tail effect to the spectrum. Peaks in optical conductivity can be observed, which can be related to interband transitions. The SPR reflectance curve based on the Kretschmann configuration has further been simulated.

Keywords Density functional theory; Optical conductivity; Surface plasmon resonance

\section{INTRODUCTION}

Scientific interests in the plasmonic materials have been growing exponentially, mainly due to the diversity of applications, including biosensing, bioimaging, biological detection, and environmental monitoring [1]. Several studies have been conducted to explore the plasmonic performance of various metals. Surface plasmon resonance (SPR) refers to a condition where there is a resonance between evanescent waves with surface plasmon waves that propagate along with the metal-dielectric interface [2]. The resonance condition depends on the sensing layer, which is represented by a dielectric constant, in which the macroscopic electronic responses of a material can be fully described by the complex dielectric constants.

Transition metals (TMs), especially noble metals, are plasmonic materials because they tend to have large plasma frequencies and high electrical conductivity [3]. Efforts to study the optical properties of transition metals in terms of optical conductivity, dielectric constant, or optical constant have been increasingly conducted for a few decades, both from experimental [4-18] and theoretical [6, 18-24] sides. For instance, Johnson and Christy reported their experimental values of the optical constants for copper, silver, and gold in the spectral range of $0.5-6.5 \mathrm{eV}$ and concluded that the interband absorption dominated in the visible and ultraviolet regimes [4]. They also determined the values of the optical constants of iron, cobalt, and nickel [5]. On the theoretical counterparts, studies on the absorptive part of optical conductivity have been carried out by using the density functional theory (DFT) approach applying the local-density approximation (LDA) on Fe and Ni [19] which only involves interband transitions but the results match well the experiments. Subsequently, Uba et al. applyed the generalized gradient approximation (GGA) approximation with Hubbard energy correction to improve the description 
of the optical conductivity spectra of noble metal [18].

To provide a systematic analysis on the SPR-related optical properties for the late series of transition metals, here, we studied the optical conductivities and dielectric constants of 3d (Fe, Ni, Co, Cu), 4d (Ru, Rh, Pd, Ag), and 5d (Os, Ir, $\mathrm{Pt}, \mathrm{Au}$ ) metals by using the DFT method. Next, by using the computed values, we calculated the reflectance of the SPR curve on the three-layer system in the Kretschmann configuration.

\section{COMPUTATIONAL METHODS}

\section{A. DFT calculation}

Self-consistent DFT calculations were performed as implemented in the full-potential linearized augmented plane wave (FLAPW) method [25-27]. The GGA was used for the exchange-correlation potential [28]. The electronic wave function was expanded on a plane-wave basis set by a kinetic energy cutoff of 3.9 a.u. $^{-1}$ and an angular momentum $l_{\max }=8$ inside the muffin-tin spheres. The number of the $k$-points in the first full Brillouin zone was set to $70 \times 70 \times$ 70. Crystal structures and lattice constants of all systems were adopted from experimental structures available in Ref. 29. In metals, the optical spectrum is relatively insensitive to small changes in the lattice parameters [30], and therefore further volume optimization was not carried out.

The optical conductivity tensor, $\sigma_{\alpha \beta}$, for metal can be separated into two terms: the interband and intraband contributions. The interband contribution was obtained from the Kubo formula [31, 32]. The Drude model has been used to combine the intraband contribution with the optical conductivity. The electron relaxation time parameter $\gamma_{\mathrm{D}}\left(=1 / \tau_{\mathrm{D}}\right)$ in the Drude model for the intraband transition were optimized by using a least square method, while the plasma frequency $\omega_{\mathrm{p}}$, directly related to the momentum operator, $-i h \nabla$, was deduced from our DFT calculations [33]. The dielectric tensor $\varepsilon_{\alpha \beta}$ is related to the optical conductivity tensor through the following equation [22];

$$
\varepsilon_{\alpha \beta}=\delta_{\alpha \beta}+\frac{4 i \pi}{\omega} \sigma_{\alpha \beta}(\omega)
$$

Here, $\delta_{\alpha \beta}$ is the Kronecker delta.

\section{B. SPR calculation}

Three-layer systems in the Kretschmann configuration, commonly used in experiments to excite surface plasmon waves, have been used in our modeling. The first layer in this configuration was a prism with dielectric constant $\varepsilon_{1}$, the second layer was a metal with dielectric constant $\varepsilon_{2}$ and thickness $d$, and the third layer was air with dielectric constant $\varepsilon_{3}$. The reflectance of the system was calculated through the equation:

$$
R=\frac{r_{12}+r_{23} \exp \left(2 i k_{2 z} d\right)}{1-r_{12} r_{23} \exp \left(2 i k_{2 z} d\right)}
$$

where

$$
\begin{aligned}
& r_{12}=\frac{k_{1 z} \varepsilon_{2}-k_{2 z} \varepsilon_{1}}{k_{1 z} \varepsilon_{2}+k_{2 z} \varepsilon_{1}}, \\
& r_{23}=\frac{k_{2 z} \varepsilon_{3}-k_{3 z} \varepsilon_{2}}{k_{2 z} \varepsilon_{3}+k_{3 z} \varepsilon_{2}},
\end{aligned}
$$

and

$$
\begin{gathered}
k_{i z}=\left(\varepsilon_{i} \frac{\omega^{2}}{c^{2}}-k_{z}\right)^{1 / 2}, \\
k_{x}=\frac{\omega}{c} \sqrt{\varepsilon_{1}} \sin \theta .
\end{gathered}
$$

Here, $r_{12}$ and $r_{23}$ are the reflection coefficients at the prism-metal interface and the metal-air interface, respectively; $k_{i z}$ is the $z$ component of the wavevector in the medium- $i ; k_{x}$ is the $x$ component of wavevector in the prism; $\omega$, $c$, and $\theta$ is the energy range, the velocity of light, and the incident angle, respectively.

In the simulations, a N-BK7 (SCHOTT) glass was used as the prism substrate for all configurations. The dielectric constant of prism $\varepsilon_{1}$ and air $\varepsilon_{3}$ obtained from https://refractiveindex.info/. The complex dielectric constant of the metal $\varepsilon_{2}=\varepsilon^{\prime}+i \varepsilon^{\prime \prime}$ was taken from our calculation results. The optimum thickness of the metal layer and the incident angle were chosen so that the reflectance had a minimum dip, shown in Table 1. The incident light was varied from 0 to $6 \mathrm{eV}$ selected where it was in the optical region $(1.65-3.26 \mathrm{eV})$

\section{RESULTS AND DISCUSSION}

\section{A. Optical properties of transition metals}

We start by discussing the optical conductivities, $\sigma_{x x}$, which include the interband and intraband contributions. Figure 1 shows the diagonal part of optical conductivity, compared to the experimental data. It is visible that the general features of the calculated optical conductivities reproduce reasonably well to the experiments. Peaks in the spectrum of the absorptive parts of the conductivity tensor $\sigma_{1 x x}$ have also been obtained in our calculations, resembling

Table 1: Parameters used in the simulation.

\begin{tabular}{lcccccccccccc}
\hline & $\mathrm{Fe}$ & $\mathrm{Co}$ & $\mathrm{Ni}$ & $\mathrm{Cu}$ & $\mathrm{Ru}$ & $\mathrm{Rh}$ & $\mathrm{Pd}$ & $\mathrm{Ag}$ & $\mathrm{Os}$ & $\mathrm{Ir}$ & $\mathrm{Pt}$ & $\mathrm{Au}$ \\
\hline Thickness $(\mathrm{nm})$ & 70 & 40 & 50 & 25 & 20 & 10 & 20 & 35 & 25 & 25 & 10 & 30 \\
Incidence angle $\left(^{\circ}\right)$ & 50 & 50 & 50 & 44 & 50 & 50 & 50 & 44 & 50 & 50 & 50 & 44 \\
\hline
\end{tabular}


those measured in experiments. These peaks can be attributed to an interband transition from just below the Fermi level to the unoccupied density of states (DOS).

Starting with $\mathrm{Fe}, \mathrm{Co}$, and $\mathrm{Ni}$, our calculation results have the same trend and only slightly differ in amplitude, especially for $\mathrm{Fe}$ and Co. The peak of the experimental spectrum of $\mathrm{Fe}$ is located at $2-3 \mathrm{eV}$, while our calculation nicely obtained the peak at around $3 \mathrm{eV}$. For Co, the experimental data have peaks around 1 and $2.1 \mathrm{eV}$. Likewise, in our results, the spectrum has a wide peak at $2.2 \mathrm{eV}$. At 4.5-6 eV, our calculation results match the experimental results. In the case of $\mathrm{Ni}$, the Drude contribution is very dominant at the low energy, resulting in a tail below $1 \mathrm{eV}$. Our calculation results have the same trend, but the peak shifts to higher energy. Our calculation results are similar to those in Refs. 19 and 20.

In the last considered $3 \mathrm{~d}$ metal in this work, $\mathrm{Cu}$, the contribution of the Drude model is very dominant at low energy levels. The calculation results have the same trend as the experimental results. The spectral shape of the experimental spectra are reproduced by the GGA calculation. However, the position of the calculated edge of the optical conductivity are shifted toward smaller energies as compared to the experimental data. This edge position shift can be confirmed from the partial $\sigma_{1 x x}$ spectra for the transitions from the uppermost $\mathrm{d}$ states (band 5) into sp-like states (band 6) just above the Fermi level, especially in the $\mathrm{X}$ and L symmetry points. Similar situations have also been found for $\mathrm{Ag}$ and $\mathrm{Au}$ from the $4 \mathrm{~d}$ and $5 \mathrm{~d}$ metals, respectively. In $\mathrm{Ag}$, the position of the edge and the peak of the spectrum results from two transitions, from band 5 (the highest $\mathrm{d}$ states) to band 6 (sp-like states) and from band 6 (sp-like states) to band 7 (sp-like states). In Au, the onset of the edge and the position of the first peak, due to transitions from band 5 (the highest $d$ states) into band 6 (sp-like states). The salient features of the calculated results at 1.7, 2.9 and 1.8 $\mathrm{eV}$ for $\mathrm{Cu}, \mathrm{Ag}$, and $\mathrm{Au}$, respectively, is observed. In $\mathrm{Au}$, intraband contributions have a significant effect on low energy which indicates that an optimized Drude model might be crucial [21].

In other atoms, namely $\mathrm{Ru}, \mathrm{Rh}, \mathrm{Pd}, \mathrm{Os}$, Ir, and $\mathrm{Pt}$, our results are also similar to experimental data. In $\mathrm{Ru}$, the dip at the low energy was observed in our calculations and matched with Ref. 6 . The first peak of the spectrum shifts to

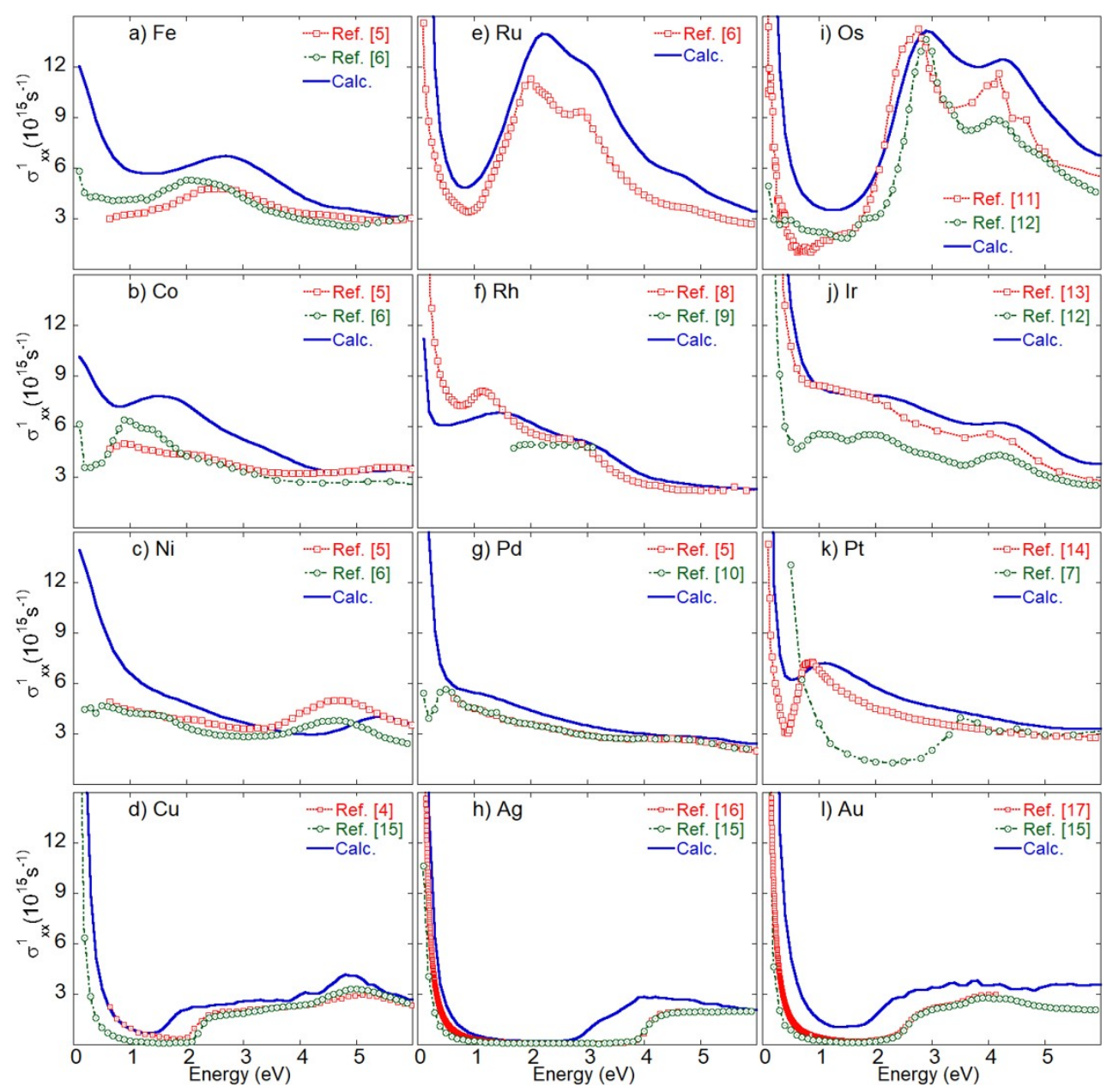

Figure 1: Calculated diagonal optical conductivity (solid lines) for transition metals atom. Lines drawn by red squares and green circles are the experimental data collected from literature; Fe [5, 6], $\mathrm{Co}[5,6], \mathrm{Ni}[5,6], \mathrm{Cu}[4,15], \mathrm{Ru}[6], \mathrm{Rh}[8,9], \mathrm{Pd}[5,10], \mathrm{Ag}[15,16], \mathrm{Os}[11,12]$, Ir [12, 13], Pt [7, 14], and Au [15, 17]. 


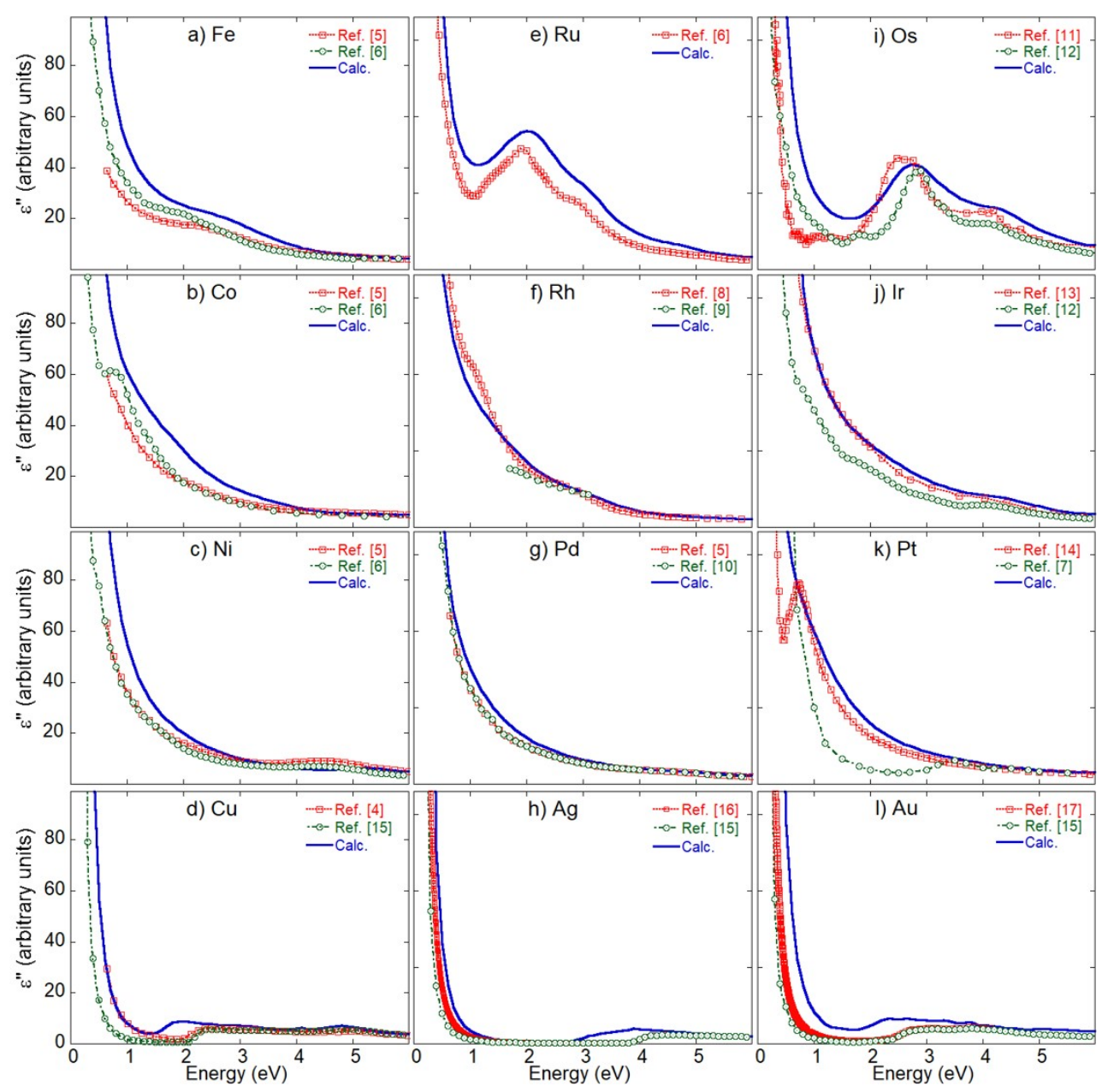

Figure 2: Comparison of the imaginary part of dielectric constants for transition metals atom. Notations are the same as Figure 1.

the high energy of $2.1 \mathrm{eV}$, and the second peak at $3 \mathrm{eV}$ is observed in our results. Likewise, for Os, the dip on the spectra of $\sigma_{1 x x}$ also appears at low energy. The first peak at 3 $\mathrm{eV}$ and the second peak at $4.2 \mathrm{eV}$ match the experimental data in Ref. 12. In Ir, our calculations are very close to Ref. 13. In Pt, the dip at low energy is not as sharp as Ref. 14. Even so, our calculations on $\mathrm{Ru}$, Ir, and Pt match the results of calculations on Ref. 22 based on the LDA.

The dielectric constant is calculated from optical conductivity through Eq. (1). The imaginary part of the dielectric constant, $\varepsilon^{\prime \prime}$, is shown in Figure 2. All calculations also match the experimental results in the reference. The imaginary part of the dielectric constant is related to energy absorption. At the low energy, our calculation results confirm that only noble metals have $\varepsilon^{\prime \prime} \approx 0$. Metallic high reflectivity was observed at $1-1.5,1-3$, and $1-2 \mathrm{eV}$ for $\mathrm{Cu}$, $\mathrm{Ag}$, and $\mathrm{Au}$, respectively. Compared to other atoms, $\mathrm{Ru}$ and Os also have dips in the low energy range so that the peak of spectra $\varepsilon^{\prime \prime}$ can be observed. Except for noble metals, transition metals show high energy losses at 1-2 eV.

The results of the calculation of the real part of dielectric constant, $\varepsilon^{\prime}$, are shown in Figure 3. Almost all calculations are in good agreement with the experiment data. We get the zero crossings of $\varepsilon^{\prime}$, which represents a bulk plasmon energy. The presence of a bulk plasmon in the optical region would facilitate the excitation of surface plasmons in these materials. In Ru and Os, the bulk plasmon can be observed at two points but shifts slightly to the higher energy compared to the reference. In noble metal, zero crossings of $\varepsilon^{\prime}$ on $\mathrm{Cu}$ at low energy are not observed. In $\mathrm{Ag}$, the zero crossings of $\varepsilon^{\prime}$ first shift to the lower energy at $3 \mathrm{eV}$, then at more than $4 \mathrm{eV}$, several bulk plasmon values appear. In $\mathrm{Au}$, there are multiple zero crossings of $\varepsilon^{\prime}$ between $3.5-4.4 \mathrm{eV}$. In other atoms, zero crossings of $\varepsilon^{\prime}$ occur in energy more than $6 \mathrm{eV}$.

\section{B. SPR simulation}

To find out the material characteristics in the SPR application, we calculated the reflectance based on Eq. (2) as shown in Figure 4. In the optical region, the curves that have dips are $\mathrm{Cu}, \mathrm{Ag}, \mathrm{Au}, \mathrm{Os}$, and $\mathrm{Ru}$. We can show that the noble metal (i.e., $\mathrm{Cu}, \mathrm{Au}$, and $\mathrm{Ag}$ ) has the optimum dip with a small full width at half maximum (FWHM). Ru and Os also have dipped but the FWHM is wide. The dip shows the resonance condition between the surface plasmon wave and the 


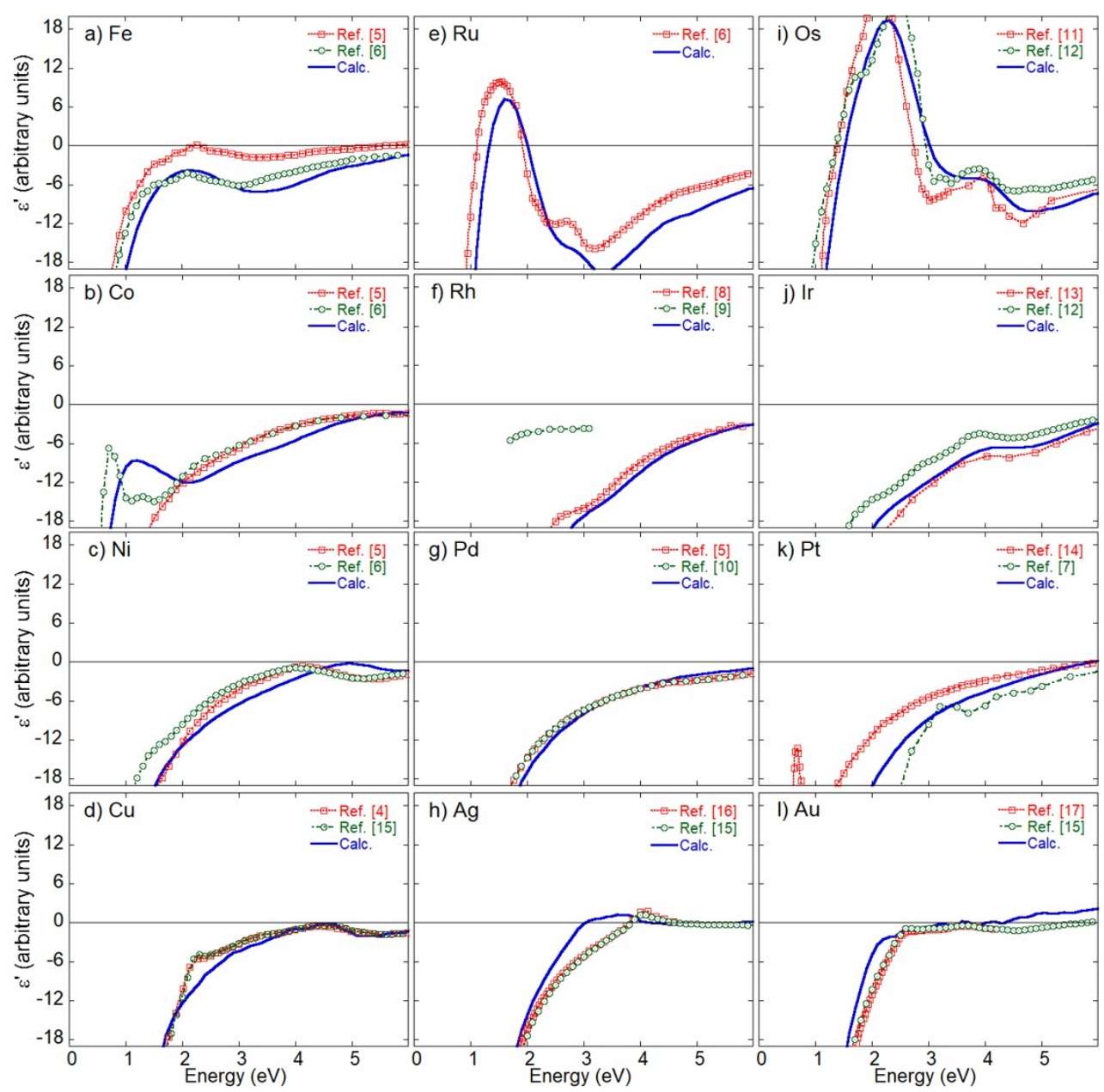

Figure 3: Comparison of the real part of dielectric constants for transition metals atom. Notations are the same as Figure 1.

evanescent wave.

Our simulation curve is similar to the reference in thin layers using the Kretschmann configuration. For $\mathrm{Cu}$, the reflectance calculation shifts to the left, indicating the difference in the thickness of $\mathrm{Cu}$ used, which is 25 and $43.5 \mathrm{~nm}$ for our calculations and Ref. 34, respectively. In Au and Ag, the curve also shifts to lower energy than the reference curve caused by differences in the prism material used, namely an ultraviolet fused quartz and $\mathrm{a} \mathrm{CaF}_{2}$ glass for Refs. 35 and 36 , respectively. The difference of the prism material indicates a different refractive index, which is sensitive to reflectance in the system.

\section{CONCLUSIONS}

Using the first-principles calculations, we calculated the optical conductivity and dielectric constants in transition metals. The contribution of the intraband transition gives a tail effect on the calculation results for low energy. At 0-6 $\mathrm{eV}$, the peaks of the spectrum in the optical conductivity are

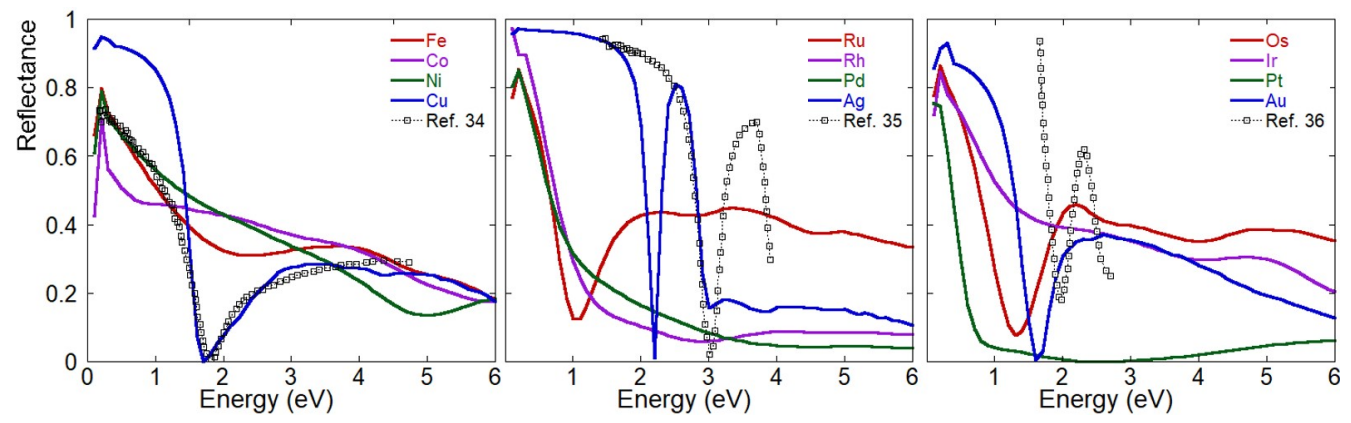

Figure 4: SPR reflectance curves as a function of the energy. Lines drawn by black squares are the experimental data from literature; $\mathrm{Cu}$ [34], $\mathrm{Ag}[35]$, and $\mathrm{Au}[36]$. 
caused by the interband transition. In the optical range, the results of the dielectric constant are in good agreement with the experimental data. We also demonstrated the SPR reflectance curve in a three-layer system in the Kretschmann configuration. In the noble metal, the simulation curve matches the references.

\section{Acknowledgments}

The work is supported by the Japan Society for the Promotion of Science (JSPS) KAKENHI Grants Nos. 15H05702 and 16K05415.

\section{Note}

This paper was presented at the 12th International Symposium on Atomic Level Characterizations for New Materials and Devices '19 (ALC '19), in conjunction with the 22nd International Conference on Secondary Ion Mass Spectrometry (SIMS-22), Miyako Messe, Kyoto, Japan, 20-25 October, 2019.

\section{References}

[1] A. A. Rifat, M. R. Hasan, R. Ahmed, and A. E. Miroshnichenko, in: Computational Photonic Sensors, edited by M. F. O. Hameed and S. Obayya (Springer, Cham, 2019) p. 203.

[2] H. Raether, Surface Plasmons on Smooth and Rough Surfaces and on Gratings (Springer, Berlin, Heidelberg, 1988).

[3] P. R. West, S. Ishii, G. V. Naik, N. K. Emani, V. M. Shalaev, and A. Boltasseva, Laser Photonics Rev. 4, 795 (2010).

[4] P. B. Johnson and R. W. Christy, Phys. Rev. B 6, 4370 (1972).

[5] P. B. Johnson and R. W. Christy, Phys. Rev. B 9, 5056 (1974).

[6] S. Adachi, The Handbook on Optical Constants of Metals (World Scientific, 2012).

[7] W. S. M. Werner, K. Glantschnig, and C. Ambrosch-Draxl, J. Phys. Chem. Ref. Data 38, 1013 (2009).

[8] J. H. Weaver, C. G. Olson, and D. W. Lynch, Phys. Rev. B 15, 4115 (1977).

[9] D. P. Arndt, R. M. A. Azzam, J. M. Bennett, J. P. Borgogno, C. K. Carniglia, W. E. Case, J. A. Dobrowolski, U. J. Gibson, T. Tuttle Hart, F. C. Ho, V. A. Hodgkin, W. P. Klapp, H. A. Macleod, E. Pelletier, M. K. Purvis, D. M. Quinn, D. H. Strome, R. Swenson, P. A. Temple, and T. F. Thonn, J. Appl. Opt. 23, 3571 (1984).

[10] A. Borghesi and A. Piaggi, in: Handbook of Optical Constants of Solids, Vol. 2, edited by E. D. Palik (Academic, Boston, 1991) p. 469.

[11] V. V. Nemoshkalenko, V. N. Antonov, VI., N. Antonov, M. M. Kirillova, A. E. Krasovskii, and L. V. Nomerovannaya, J. Exp. Theor. Phys. 63, 115 (1986).

[12] D. W. Lynch and W. R. Hunter, in: Handbook of Optical Constants of Solids, Vol. 1, edited by E. D. Palik (Academic Press, Boston, 1985) p. 275

[13] L. Yan and J. A. Woollam, J. Appl. Phys. 92, 4386 (2002).

[14] J. H. Weaver, Phys. Rev. B 11, 1416 (1975).

[15] S. Babar and J. H. Weaver, Appl. Opt. 54, 477 (2015).

[16] H. U. Yang, J. D'Archangel, M. L. Sundheimer, E. Tucker, G. D. Boreman, and M. B. Raschke, Phys. Rev. B 91, 235137 (2015).
[17] R. L. Olmon, B. Slovick, T. W. Johnson, D. Shelton, S.-H. Oh, G. D. Boreman, and M. B. Raschke. Phys. Rev. B 86, 235147 (2012).

[18] L. Uba, S. Uba, and V. N. Antonov, Phys. Rev. B 96, 235132 (2017).

[19] P. M. Oppeneer, T. Maurer, J. Sticht, and J. Kübler, Phys. Rev. B 45, 10924 (1992).

[20] A. Delin, O. Eriksson, B. Johansson, S. Auluck, and J. M. Wills, Phys. Rev. B 60, 14105 (1999).

[21] H. S. Sehmi, W. Langbein, and E. A. Muljarov, Phys. Rev. B 95, 115444 (2017).

[22] W. S. Choi, S. S. A. Seo, K. W. Kim, T. W. Noh, M. Y. Kim, and S. Shin, Phys. Rev. B 74, 205117 (2006).

[23] R. Lässer and N. V. Smith, Phys. Rev. B 25, 806 (1982).

[24] R. Lässer, N. V. Smith, and R. L. Benbow, Phys. Rev. B 24, 1895 (1981).

[25] E. Wimmer, H. Krakauer, M. Weinert, and A. J. Freeman, Phys. Rev. B 24, 864 (1981).

[26] K. Nakamura, T. Ito, A. J. Freeman, L. Zhong, and J. Fernandez-de-Castro, Phys. Rev. B 67, 014420 (2003).

[27] M. Weinert, E. Wimmer, and A. J. Freeman, Phys. Rev. B 26, 4571 (1982).

[28] J. P. Perdew, K. Burke, and M. Ernzerhof, Phys. Rev. Lett. 77, 3865 (1996).

[29] R. W. G. Wyckoff, Crystal Structures, Vol. 1 (Interscience Publishers, New York, 1963) p. 7.

[30] V. J. Keast, B. Zwan, S. Supansomboon, M. B. Cortie, and P. O. Å. Persson, J. Alloys Compd. 577, 581 (2013).

[31] D. L. Windt, W. C. Cash, M. Scott, P. Arendt, B. Newnam, R. F. Fisher, and A. B. Swartzlander, Appl. Opt. 27, 246 (1988).

[32] C. S. Wang and J. Callaway, Phys. Rev. B 9, 4897 (1974).

[33] H. S. Bennett and E. A. Stern, Phys. Rev. 137, A448 (1965).

[34] V. G. Kravets, R. Jalil, Y.-J. Kim, D. Ansell, D. E. Aznakayeva, B. Thackray, L. Britnell, B. D. Belle, F. Withers, I. P. Radko, Z. Han, S. I. Bozhevolnyi, K. S. Novoselov, A. K. Geim, and A. N. Grigorenko, Sci. Rep. 4, 5517 (2015).

[35] K. Takagi, S. V. Nair, R. Watanabe, K. Seto, T. Kobayashi, and E. Tokunaga, J. Phys. Soc. Japan 86, 124721 (2017).

[36] P. Arora, E. Talker, N. Mazurski, and U. Levy, Sci. Rep. 8, 9060 (2018).

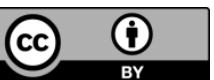

All articles published on e-J. Surf. Sci. Nanotechnol. are licensed under the Creative Commons Attribution 4.0 International (CC BY 4.0). You are free to copy and redistribute articles in any medium or format and also free to remix, transform, and build upon articles for any purpose (including a commercial use) as long as you give appropriate credit to the original source and provide a link to the Creative Commons (CC) license. If you modify the material, you must indicate changes in a proper way.

Published by The Japan Society of Vacuum and Surface Science 